\title{
Useful Work and Gibbs Energy
}

\author{
Nikolai Bazhin
}

Additional information is available at the end of the chapter

http://dx.doi.org/10.5772/50119

\section{Introduction}

Devices for performing chemical reactions are widely used to produce heat and work. Heat, in turn, produces work, e.g., in the form of electric energy in the so-called heat engines. It is the well-known fact that the efficiency of heat engines is restricted by Carnot principle. Therefore, it is generally recognized that heat cannot be fully converted to work. The efficiency of electric energy production due to the burning of fossil fuel of various kinds varies from 30 to $50 \%$.

On the other hand, there are galvanic and fuel cells whose efficiency can reach theoretically unity if it implies the ratio between the electric energy produced and the value of a change in Gibbs energy during chemical reactions occurring in a cell. These devices operate at constant temperature and pressure. It is concluded then that the devices, similar to a galvanic cell, cannot work at constant and uniform temperature according to the principle of heat engine. These devices assumed to operate only due to the direct transformation of chemical reaction energy, described by a change in the Gibbs energy, into work [1]. This viewpoint causes, however, numerous contradictions. The goal of this work is to analyze in detail the mechanism of useful work and heat production in chemical systems functioning at constant temperature and pressure.

\section{Fundamental functions}

In this Section, the fundamental functions of thermodynamics will be characterized, as the fundamental functions play the important role in the description of the process of the energy transformation. This notion includes four functions, i.e., internal energy, enthalpy, Helmholtz energy, and Gibbs energy. All of them are the state functions of energy dimension. It is generally believed that the value with energy dimension describes energy but this is by no means always the case. Below, the fundamental functions will elucidate whether these are energy values or not. 


\subsection{Internal energy}

According to IUPAC [2], "internal energy $U$ is the quantity the change in which is equal to the sum of heat, $q$, brought to the system and work, $w$, performed on it, $\Delta U=q+w$ ". Because of various transformations, the internal energy can be converted to other kinds of energy. However, the initial quantity of internal energy should be conserved due to the law of energy conservation. Conservation is the most important characteristic of energy. Hence $U$ is energy.

\subsection{Enthalpy}

According to IUPAC, "enthalpy, $H=U+p V$ is the internal energy of a system plus the product of pressure and volume. Its change in a system is equal to the heat, brought to the system at constant pressure" [2]. It is worth noting that the change in the value describing energy always corresponds to the change in energy and not only in some special cases. Generally, a change in enthalpy may be inconsistent with the change in real physical values. Let us consider, e.g., the process of ideal gas heating at constant volume. The quantity of heat, taken in by ideal gas from the heat bath, $q$, equivalently changes only the internal gas energy, $\Delta U=q$, and simultaneously causes changes in gas enthalpy, $\Delta H=\Delta U+\Delta p V>q$. However, this change fails to reflect the changes in any physically significant values. Thus, the enthalpy is not energy but a function of state having the dimension of energy. It is easier and more correct to assume that the enthalpy is the part of a calculating means used to describe thermodynamic processes.

\subsection{Helmholtz energy}

According to IUPAC, "Helmholtz energy (function), $A$, is the internal energy minus the product of thermodynamic temperature and entropy: $A=U$ - TS. It was formerly called free energy" [2]. Let us see why this quantity was called free energy. According to the first law [3]

$$
\Delta U=q+w,
$$

where $q$ is the heat, entering the system, and $w$ is the total work performed on the system by the surroundings. Usually, the total work is given as the sum of two terms: expansion work $(-p \Delta V)$ and so-called useful work $\left(w_{\text {useful }}\right)$

$$
w=-p \Delta V+w_{\text {useful }} .
$$

If the process occurs at constant volume, the expansion work is absent and $w$ describes the useful work. In the case of reversible process [3]

$$
q=T \Delta S
$$

Thus, eq. (1) is of the form in the case of reversible process 


$$
w_{\text {useful }}=\Delta U-T \Delta S=\Delta(U-T S)=\Delta A,
$$

where $\Delta A$ is the change in Helmholtz energy at constant temperature and volume. Since the term "energy" means the capacity of the system to perform work, from eq. (4) it is formally concluded that $A$ is the energy (but in this case eq. (4) can have the second explanation $-A$ is the numerical characteristic of work and not the work itself). Further, from eq. (4) it was concluded that only the part of internal energy $U$ minus TS can be used to produce work. Therefore, the TS quantity was called "bound energy" and (U - TS) - "free energy". The meaning of these notions will be considered in more detail using the Gibbs energy as an example because it is more often used in chemical applications.

\subsection{Gibbs energy}

According to IUPAC [2], "Gibbs energy (function), $G=(H-T S)$, is the enthalpy minus the product of thermodynamic temperature and entropy. It was formerly called free energy or free enthalpy". The reasons for the appearance of the term "Gibbs energy" are similar to those discussed when considering the Helmholtz energy except for the fact that the Gibbs energy describes the reversible useful work performed at constant temperature and pressure. This is readily observed by substituting eq. (3) and eq. (2) into eq. (1) with regard to $V \Delta p=0$

$$
w_{\text {useful }}=\Delta H-T \Delta S=\Delta(H-T S)=\Delta G,
$$

where $\Delta G$ is the change in Gibbs energy at constant temperature and pressure in the reversible process.

Unfortunately, the word "energy" as defined by IUPAC for a Gibbs energy (and also for a Helmholtz energy), causes great confusion. The Gibbs energy $G=H-T S$ consists of two terms, the enthalpy and the entropy one. The origin of both of the terms is quite different despite the same dimension. The enthalpy, considered above, is not energy.

Consider now the problem of $T S$ nature. In the case of the reversible process $T \Delta S=q$, but in the case of the irreversible process $T \Delta S>q$ and additional contributions to $T \Delta S$ can arise without change in energy. For example, it is well known the increase of the entropy in the process of ideal gas expansion in vacuum without heat consumption $(q=0)$.

Let us consider another example. Let the ideal gas-phase system involves a spontaneous process of the monomolecular transformation of substance A into B. As suggested a change in enthalpy tends to zero in this reaction. Thus, the internal energy, temperature, pressure, and volume will not undergo changes in this process. However, the entropy will increase due to the entropy of the mixing, because the entropy is a function of state. The value of the TS product will increase accordingly. However, the energy and even the bound energy cannot arise from nothing, whereas the entropy, being a function of state, can increase thus reflecting a change in system state without any changes in the internal system energy. Therefore, the TS term is not the energy, which also implies the absence of the term "bound" energy. 
Since neither enthalpy nor TS are the energy quantities, the difference between them cannot represent energy. Thus, $G$ cannot represent energy precisely in terms of this notion. Note that in the irreversible process, occurring at constant temperature and pressure, the Gibbs energy decreases and thus, is not conserved. This is readily demonstrated by e.g., the aforementioned example of a monomolecular transformation of substance A into B. Thus, conservation, as the most important criterion for energy quantity, fails for the Gibbs energy. It is concluded then that the Gibbs energy is not energy [4] but a function of state. In this regard, the Gibbs energy does not differ from heat capacity. The notions of the non-existing in reality quantities of "free energy" and "bound energy" cause only confusion and are, at present, obsolete [5].

Nevertheless, the notions that the Gibbs energy is the energy and thus, obeys conservation laws, prove to be long-lived, which causes erroneous interpretation of a number of processes some of which are of paramount importance.

Let us consider now the reaction of adenosine triphosphate (ATP) hydrolysis in water solution which is of great concern in biochemistry

$$
\mathrm{ATP}+\mathrm{H}_{2} \mathrm{O}=\mathrm{ADP}+\mathrm{Pi}
$$

Under the standard conditions [6] $\Delta_{\mathrm{r}} G^{\mathrm{o}}=-7, \Delta_{\mathrm{r}} H^{\mathrm{o}}=-4 \mathrm{kcal} \cdot \mathrm{mol}^{-1}$. According to D. Haynie [6, p. 143], "measurement of the enthalpy change of ATP hydrolysis shows that $\Delta H^{\circ}=-4$ $\mathrm{kcal} \cdot \mathrm{mol}^{-1}$. That is, the hydrolysis of one mole of ATP at $25{ }^{\circ} \mathrm{C}$ results in about $4 \mathrm{kcal} \cdot \mathrm{mol}^{-1}$ being transferred to a solution in the form of heat and about $3 \mathrm{kcal} \cdot \mathrm{mol}^{-1}$ remaining with ATP and Pi in the form of increased random motion."

The heat of $4 \mathrm{kcal} \cdot \mathrm{mol}^{-1}$ is actually released into solution due to hydrolysis. Unfortunately, it is then assumed that the Gibbs energy is conserved which makes his difference in $\Delta_{\mathrm{r}} H^{\mathrm{o}}$ and $\Delta_{\mathrm{r}} G^{\mathrm{o}}$ of $3 \mathrm{kcal} \cdot \mathrm{mol}^{-1}$ be located on the degrees of freedom of product molecules. However, in this connection, the product molecules could appear in the non-equilibrium excited states and transfer this energy to solvent molecules which would result in the emission of $7 \mathrm{kcal} \cdot \mathrm{mol}^{-1}$ rather than $4 \mathrm{kcal} \cdot \mathrm{mol}^{-1}$ which contradicts the experiment. There are no additional $3 \mathrm{kcal} \cdot \mathrm{mol}^{-1}$ on the degrees of freedom of ATP and Pi because the Gibbs energy is not conserved.

\subsection{Conclusions}

Thus, among the four quantities, that claims to be called energy quantity, only the internal energy deserves this name. The other functions, i.e., enthalpy $H$, Helmholtz energy $A$, and Gibbs energy $G$ are the parts of a mathematical apparatus for calculating various quantities, such as useful work, equilibrium constants, etc. This also means that the useful work is only calculated by using functions $A$ and $G$, but cannot arise from the change in either the Helmholtz or the Gibbs energy. The physical nature of the work performed should be considered separately. Since the Helmholtz energy and the Gibbs energy are not energies, then, to avoid misunderstanding, it is better to exclude the word "energy" from the name of corresponding functions and to use the second variant of the name of these functions according to IUPAC: a Helmholtz function and a Gibbs function [7]. 


\section{Direct conversion of chemical reaction energy to useful work}

This Section is devoted to the discussion of the generally accepted theory of the direct conversion of energy $[1,8]$ produced by chemical reaction to useful work. For simplicity, hereafter exergonic $\left(\Delta_{\mathrm{r}} G<0\right)$ and exothermic $\left(\Delta_{\mathrm{r}} H<0\right)$ reactions will be considered.

The useful work of the chemical reaction occurring at constant temperature and pressure in the reversible conditions can be calculated through the change in the Gibbs function (5). When the interest is the useful work performed by the system in the environment $\left(-w_{\text {useful }}\right)$, then

$$
-w_{\text {useful }}=-\Delta_{\mathrm{r}} H+T \Delta_{\mathrm{r}} S=-\Delta_{\mathrm{r}} G
$$

From eq. (6) it follows formally that the useful work of the reversible system in the environment is the sum of the enthalpy member $\left(-\Delta_{\mathrm{r}} H\right)$ and the entropy member $T \Delta_{\mathrm{r}} S$. In this connection it is interesting to discuss the various situations which arise in dependence on the relation between $\left(-\Delta_{\mathrm{r}} H\right)$ and $T \Delta_{\mathrm{r}} S$.

From eq. (6) it follows, that for $\Delta_{\mathrm{r}} S>0$ the useful work in the environment exceeds $\left(-\Delta_{\mathrm{r}} H\right)$ : $-w_{\text {useful }}>-\Delta_{\mathrm{r}} H$. Therefore, the system must drag the thermal energy from the environment in the volume $T \Delta_{\mathrm{r}} S$ to perform useful work. What is the physical reason for thermal energy consumption? Why does the system consume thermal energy of volume $T \Delta_{\mathrm{r}} S$ neither more or less? How can two different contributions produce the same useful work?

The second case of $\Delta_{\mathrm{r}} S<0$ is also of interest. In this case $-w_{\text {useful }}<-\Delta_{\mathrm{r}} H$ and the system must evolve the part of reaction heat to the environment. Why cannot the system use the total reaction heat for useful work production if this energy is at its disposal? Why can the system transfer energy of volume $T \Delta_{\mathrm{r}} S$ and neither more or less to the environment?

The third case is $\Delta_{\mathrm{r}} H=0$. Here the system can use only the thermal energy of the environment to produce useful work.

In the fourth case $\Delta_{\mathrm{r}} S=0$ and the system performs work formally due to the reaction heat $\left(-\Delta_{\mathrm{r}} H\right)$ without exchanging thermal energy with the environment. But it is not the case.

As mentioned in the Introduction, at present, it is generally accepted that the high efficiency of reversible devices is inconsistent with the notions that heat can be used to produce work and that such devices realize the direct conversion of chemical system energy into work. But below it will be shown that in all the cases, the useful work results from the heat of volume $\Delta_{\mathrm{r}} G$ dragged from the environment.

\section{A mechanism of useful work production - A Van't Hoff Equilibrium Box}

In this Section, it will demonstrate the mechanism of producing useful reversible work which involves no notions of the direct conversion of reaction energy into useful work. To this end, let us consider a Van't Hoff equilibrium box (VHEB) [9 - 11]. A thermodynamic 
system must provide realization of the reversible process. This means that all changes in the system are infinitely slow at infinitely minor deviation from equilibrium.

It is assumed that in the system the following reaction occurs

$$
\sum_{i} v_{i} \mathrm{~A}_{i}=\sum_{j} v_{j} B_{j}
$$

where $\mathrm{A}_{i}$ are the reagents and $\mathrm{B}_{j}$ are the products. The reaction takes place in the reactor (Fig. 1) where the reagents and products are in equilibrium. The chemical process is afforded by reservoirs with reagents and products contained in the system. For simplicity, the reagents and products are assumed to be in standard states. The system should have instruments to transport both the reagents from standard vessels to reactor and the products from reactor to standard vessels. In addition, the system should have tools to perform work, because the reversible process must be followed by reversible work production. The instruments and tools for performing work can be used together. The reactor, transporting instruments, and tools for performing work can be placed either separately or together. To provide constant and uniform temperature, it is necessary to locate the system, including reactor, standard vessels, transporting instruments, and tools in a thermostat, which can also imply the environment of constant temperature.

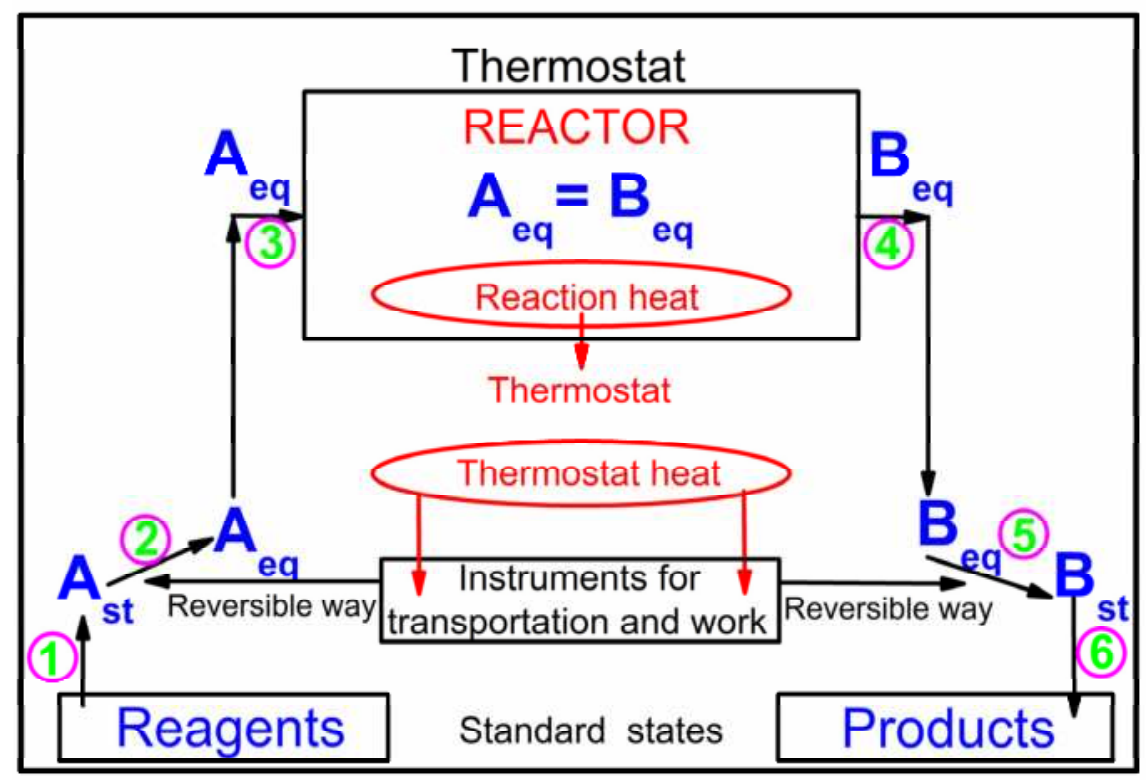

Figure 1. Production of useful work and heat in a closed reversible system. $A_{s t}\left(B_{s t}\right)$ indicate the reagents $\mathrm{A}_{i}$ (products $\mathrm{B}_{j}$ ) in the standard states; $\mathrm{A}_{\text {eq }}\left(\mathrm{B}_{\mathrm{eq}}\right)$ indicate the reagents $\mathrm{A}_{i}$ (products $\mathrm{B}_{j}$ ) in the equilibrium states, which correspond to the equilibrium at the reactor; the green figures in circles indicate the step numbers (see text) 
Let us consider reversible chemical process in a closed system (Fig. 1). The realization of the reversible chemical process consists in reversible transformation of the reagents to products via chemical reaction. Let us consider the closed system.

\subsection{Closed system}

The process of reversible work production includes six stages.

Step 1. A small amount of substance $A_{i}$ is removed from the vessel with reagent $A_{i}$ in the standard state upon reversible process. Gaseous substances can be removed from a standard vessel and put into a portable cylinder with pistons [11]; solid or liquid substances are placed in lock chambers.

Then, the change in the Gibbs function and the work are zero $\left(\Delta G_{1 i}=0, w_{1 i}=0\right)$ because a minor portion of substance $\mathrm{A}_{i}$ is in the same standard state as the residual reagent.

Step 2. Reagent $A_{i}$ is transformed reversibly from the standard into the equilibrium state in the reactor. For example, for ideal gas, the gas pressure will vary from a standard value to the equilibrium partial value in the reactor. In this stage, the reversible work $w_{2 i}$ is produced and $\Delta G_{2 i} \neq 0$. The work $w_{2 i}$ can be done only due to thermostat heat because there are no other energy sources in the system (reaction is in the equilibrium). This work depends on the difference in the physical states of reagent $\mathrm{A}_{i}$ in the initial and equilibrium states. All reagents $\mathrm{A}_{i}$ participate in all stages in quantities proportional to $v_{i}$. For ideal gas, the useful work is

$$
w_{2 i}=v_{i} R T \ln \left(p_{i, \mathrm{eq}} / p_{i, \mathrm{st}}\right),
$$

where $p_{i \text {,eq }}$ is the equilibrium pressure of $i$-th gas in the reactor, and $p_{i, \text { st }}$ is the pressure of $i$-th gas in a standard vessel. For gaseous components, e.g., the process of reversible gas expansion (compression) in a portable cylinder for producing the maximum useful work, must proceed to the value $p_{i, \text { eq }}$. If expansion (compression) stops at $p_{i}>p_{i, \text { eq }}$, then the inlet of gas into the reactor causes irreversible gas expansion and thus, the useful work will be less than the maximum one. When due to expansion (compression) the final pressure is less than $p_{i, \text { eq }}$, then the inlet of gas into the reactor causes the irreversible inlet of the $i$-th gas from an equilibrium mixture in the reactor to the portable cylinder, which also leads to a decrease in useful work. The solid and liquid substances can be transported by lock chambers. The pressure above either solid or liquid substances is varied from 1 bar to the value of the total equilibrium pressure in the reactor. The pressure is created using a minor portion of equilibrium reaction mixture.

The thermostat enthalpy varies as follows: $\Delta H_{2 i \text {,thermostat }}=w_{2 i}$.

Step 3. Reagent $\mathrm{A}_{i}$ is reversibly introduced into the reactor. Gaseous components are introduced into the reactor through semipermeable membranes using portable cylinders [11]; the solid or liquid ones - by means of lock chambers. Hence, $\Delta G_{3 i}=0, w_{3 i}=0$. 
The useful work production and the change in thermostat enthalpy $\left(\Delta H_{2, \text { thermostat }}\right)$ take place only at step 2:

$$
w_{2}=\Delta H_{2, \text { thermostat }}
$$

where $w_{2}=\sum_{i} w_{2 i}$ and $\Delta H_{2, \text { thermostat }}=\sum_{i} \Delta H_{2 i, \text { thermostat }}$.

The same procedure is used to bring products from the standard vessels to reactor.

Step 4. An equivalent amount of product $\mathrm{B}_{J}$ is reversibly removed from the reactor. After this step is $\Delta G_{4 j}=0, w_{4 j}=0$.

Step 5. Product $\mathrm{B}_{J}$, removed from the reactor, is reversibly transformed from the equilibrium state into the standard one to perform work $w_{5 j}$. The change in the Gibbs function is not zero, $\Delta G_{5 j} \neq 0$. The change in the thermostat enthalpy is $\Delta H_{5 j, \text { thermostat }}=w_{5 j}$.

Step 6. Product $\mathrm{B}_{J}$, removed from the reactor, is reversibly introduced into the standard vessel. In this case is $\Delta G_{6 j}=0$ and $w_{6 j}=0$.

The change in the thermostat enthalpy upon thermal energy conversion into useful work at step 5 is

$$
w_{5}=\Delta H_{5, \text { thermostat }}
$$

where and $w_{5}=\sum_{j} w_{5 j}, \Delta H_{5, \text { thermostat }}=\sum_{j} \Delta H_{5 j, \text { thermostat }}$

The change in the thermostat enthalpy at the second and fifth steps obeys the equation

$$
\Delta H_{2, \text { thermostat }}+\Delta H_{5, \text { thermostat }}=-Q_{\text {dragged }},
$$

where $Q_{\text {dragged }}$ is the heat dragged by tools.

For the reversible process, the maximal useful work is numerically equal to $\Delta_{\mathrm{r}} G$, eq. (5) and, hence, the heat dragged by tools from thermostat in the volume

$$
w_{\text {useful }}=w_{2}+w_{5}=\Delta_{\mathrm{r}} G=\Delta_{\mathrm{r}} H-T \Delta_{\mathrm{r}} S=-Q_{\text {dragged }} .
$$

The process has resulted in the useful work of the reaction, $\Delta_{\mathrm{r}} G$, but the reaction did not occur yet. To put it otherwise, reaction work was performed without reaction. Only the thermal energy of the thermostat (environment) may be the source of work. This means that the process of useful work production and the reaction itself may be temporally and spatially separated. Thus, eq. (6) numerically connects reaction parameters and the magnitude of the work. However, no reaction energy is needed to produce the work. There is no need to subdivide energy sources into reaction source $\left(-\Delta_{\mathrm{r}} H\right)$ and thermal $T \Delta_{\mathrm{r}} S$, because there is only one energy source: the thermal energy of thermostat (environment). 
For $\left(\Delta_{\mathrm{r}} S>0\right)$, the thermal energy dragged by the tools exceeds $-\Delta_{\mathrm{r}} H,\left(Q_{\text {dragged }}>-\Delta_{\mathrm{r}} H\right)$; in the case of $\left(\Delta_{\mathrm{r}} S<0\right)$, the dragged thermal energy is less than $-\Delta_{\mathrm{r}} H \quad\left(Q_{\text {dragged }}<-\Delta_{\mathrm{r}} H\right)$; in the case of $\left(\Delta_{\mathrm{r}} S=0\right)$ the energy extracted is equal to $-\Delta_{\mathrm{r}} H \quad\left(Q_{\text {dragged }}=-\Delta_{\mathrm{r}} H\right)$ and for $\left(\Delta_{\mathrm{r}} H=0\right)$ the dragged thermal energy is of volume $T \Delta_{\mathrm{r}} S\left(Q_{\text {dragged }}=T \Delta_{\mathrm{r}} S\right)$. The volume of extracted thermal energy is controlled by chemical equilibrium via $\Delta_{\mathrm{r}} G$.

Thus, the mixture in the reactor is moved off balance to be restored later. As a result, the reaction heat is emitted into the thermostat. Indeed, because of the elementary chemical act in the reactor, the energy released concentrates at the degrees of freedom of the product molecules. As the reactor temperature is constant, this energy is dissipated in the reactor and transferred to the thermostat which causes a $-\Delta_{\mathrm{r}} H$ change in thermostat enthalpy. The total change in thermostat enthalpy is

$$
\Delta H_{\text {thermostat }}=w_{2}+w_{5}-\Delta_{\mathrm{r}} H .
$$

The cycle is over. Equations $(12,13)$ can be used to calculate the total change in thermostat enthalpy

$$
\Delta H_{\text {thermostat }}=\Delta_{\mathrm{r}} G-\Delta_{\mathrm{r}} H=-T \Delta_{\mathrm{r}} S .
$$

The change in thermostat enthalpy is controlled only by reaction entropy [11].

\subsection{The main principles of reversible device functioning in useful work production}

This consideration demonstrates the main characteristics of the reversible process of useful work production at constant temperature and pressure in closed systems:

1. The useful work arises from the stage of the reversible transport of reagents from reservoir to reactor and from the stage of the reversible transport of products from the reactor.

2. The only energy source of useful work is the thermal energy of thermostat (or environment).

3. The heat released by chemical reactions is dissipated to the thermostat; the reaction heat is infinitely small in comparison with the volume of the thermostat thermal energy; therefore no reaction heat is really needed to produce work.

4. Although the useful work is produced by the cooling of one body (thermostat), the second law of thermodynamics is not violated, because the process is followed by a change in the amount of reagents and products.

5. The useful work is produced by heat exchange with thermostat (environment) according to the scheme

reaction heat $\rightarrow$ thermal thermostat energy $\rightarrow$ useful work

(scheme I)

6. The maximal useful work is equal the heat dragged by tools from thermostat.

7. Useful work depends on the difference in the concentrations of standard and equilibrium states of reagents and products. Therefore, the amount of extracted energy can be calculated via the change in the Gibbs function. 
8. There is no direct conversion of the Gibbs energy into useful work. Gibbs energy is equal numerically the thermal energy dragged by systems from the environment for doing work.

\subsection{The energy limit of chemical reactions - Open systems}

Usually, the total energy which can be produced by chemical system, is $-\Delta_{r} H$. However, this holds for closed systems only. For open systems, the case is quite different [11].

The open system is depicted in Fig. 2. As compared with the closed one (Fig. 1), the open system consists of two thermostats: the first one contains a reactor and the second one contains standard vessels with substances A and B and tools. The second thermostat can be replaced by the environment. In the process, steps 1, 2, 5, and 6 occur in the second thermostat (environment); steps 3 and 4 take place in the first one. Thus, the heat is released in the first thermostat only and the work is performed by thermal energy of the second thermostat (environment). The processes of heat and work production are spatially separated! The energy potential of the open system obeys the equation

$$
q+w_{\text {useful }}=\Delta_{\mathrm{r}} H+\Delta_{\mathrm{r}} G
$$

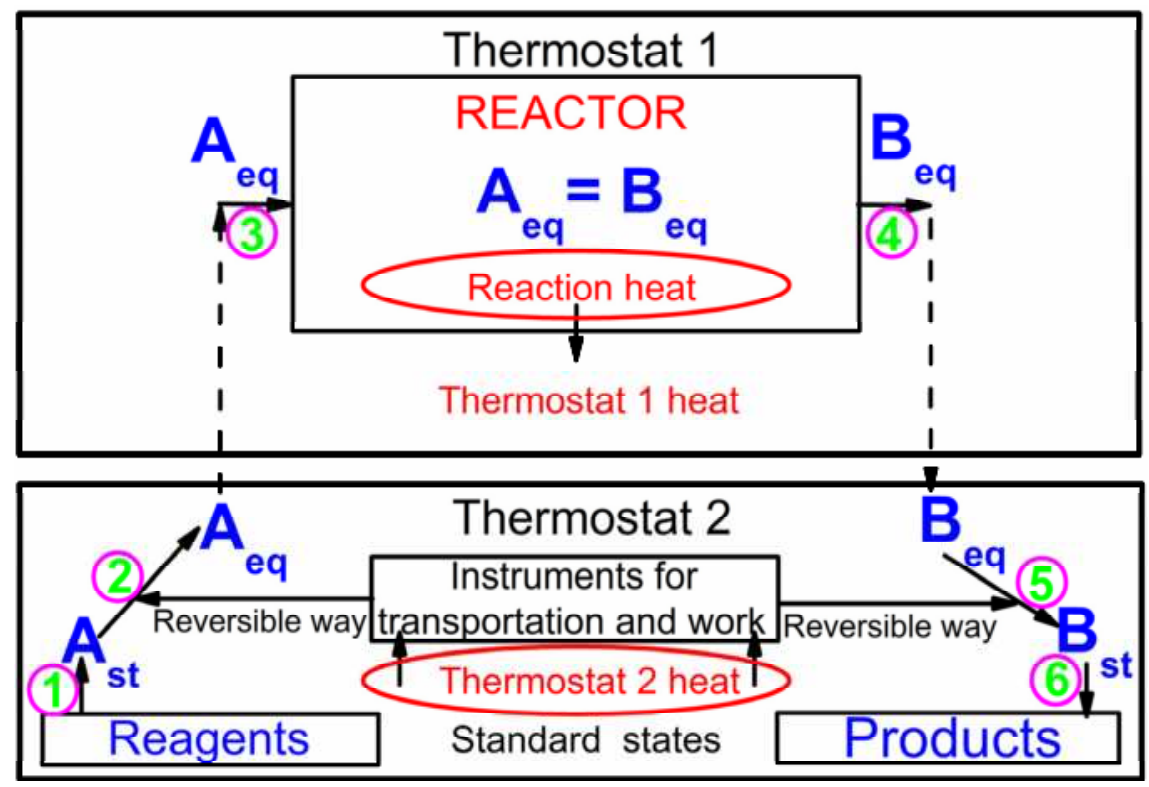

Figure 2. Production of useful work and heat in the open reversible system. The designations see in the subscription to Fig. 1

In the case of coal burning, it is possible to obtain the double total energy [11]. Thus, understanding the mechanism of useful work production in the reversible process allows us to predict an increase in the energy potential of chemical reactions in the open system. 
It is worth noting that the open system under study is not a heat pump. The heat pump consumes energy to transfer heat from a cold body to the warm one. The open system studied does not consume external energy and produces heat due to chemical reactions in one thermostat and performs work by extraction of thermal energy from the second one.

\subsection{Conclusions}

The chemical reaction heat is always released in the reversible chemical processes and passes to the environment independent of the fact whether the system produces work or not, whether it is closed or open. The discussed mechanism of useful work production in the reversible systems did not use such notions as "free energy", "bound energy", "direct Gibbs energy conversion". The useful work arises only due to the heat exchange with a thermal basin in the process, described by the scheme I. The total energy of chemical system can be high and equal to $\Delta_{\mathrm{r}} H+\Delta_{\mathrm{r}} G$.

\section{The mechanism of electric work production in a galvanic cell}

The current theory of galvanic cells $[1,3,8]$ is based on a direct transformation of the energy $\left(\Delta_{\mathrm{r}} G\right)$ of oxidation-reduction reactions into electric work. However, using VHEB as an example, It is clear that the energy of chemical reactions is first converted into the thermal energy of thermostat (environment) and then the thermal energy is extracted from the thermostat and transformed into work by means of special devices. It is assumed then that in galvanic cells, useful work is produced via the mechanism similar to the VHEB one [12, 13]. The $\Delta_{\mathrm{r}} G$ value is used to calculate electric work which does not, however, mean that the electric work is performed at the expense of the Gibbs energy, all the more it was shown that the Gibbs energy is not energy. The electric work of a galvanic cell results from the electrodes discharged. Electric charging of electrodes is caused by chemical reactions in electrodes.

The mechanism of electric energy production in galvanic cells will be solved by analyzing the behavior of one ion. But it does not denote that thermodynamics will be applied to a real single ion: thermodynamic parameters of one ion imply the averaged parameters of many ions.

\subsection{A galvanic cell}

For simplicity a Daniell cell will be considered, consisting of zinc (№1) and copper (№2) electrodes (Fig. 3). The activity of salts in solutions is denoted by $a_{1}$ and $a_{2}$, respectively. Let the cell with an open, external circuit be in equilibrium. Close now the external circuit for the moment and two electrons will transfer from the zinc to the copper electrode. The balance of the cell is distorted. Consider now the establishment of equilibrium on the zinc electrode (Fig 3). To this end, the zinc ion must leave a metallic plate and escape into the bulk. The dissolving of zinc ions is described by the change in a Gibbs function

$$
\Delta_{\mathrm{r}} G_{1}=\Delta_{\mathrm{r}} G_{1}^{\mathrm{o}}+R T \ln a_{1}
$$


where $\Delta_{\mathrm{r}} G_{1}^{\mathrm{o}}$ is a standard change in the Gibbs function upon dissolving. The ion penetrates further into solution with execution of the work $\left(w_{1 g}\right)$ in the electric field

$$
w_{1 g}=n F \Delta_{\mathrm{Met} 1}^{\mathrm{sol}} \varphi_{1}
$$

where $n$ is the number of electrons, participating in the reactions, $F$ is the Faraday constant, and $\Delta_{\text {Met1 }}^{\text {sol }} \varphi_{1}$ is the difference in potentials of solution and metal. The work described in equation (17), is the electric work spent to charge an electrode. It is performed at the extraction of the thermal energy of solution due to the absence of other energy sources in the system. Since in equilibrium, the chemical potential of ions in solution equals the chemical potential of metal, it is possible to derive the equation for electrochemical equilibrium

$$
\Delta_{\mathrm{r}} G_{1}^{\mathrm{o}}+R T \ln a_{1}+n F \Delta_{\mathrm{Met} 1}^{\mathrm{sol}} \varphi_{1}=0,
$$

which readily gives the expression for both the work performed on the first electrode and its galvanic potential [3]

$$
\begin{gathered}
w_{1 g}=n F \Delta_{\text {Met1 }}^{\mathrm{sol}} \varphi_{1}=-\Delta_{\mathrm{r}} G_{1}^{\mathrm{o}}-R T \ln a_{1}, \\
\Delta_{\mathrm{Met} 1}^{\mathrm{sol}} \varphi_{1}=-\frac{\Delta_{\mathrm{r}} G_{1}^{\mathrm{o}}}{n F}-\frac{R T \ln a_{1}}{n F} .
\end{gathered}
$$

The latter is the potential for a half-cell. Thus, the approach, based on the consideration of the behavior of one ion, provides a common expression for electrode potential.

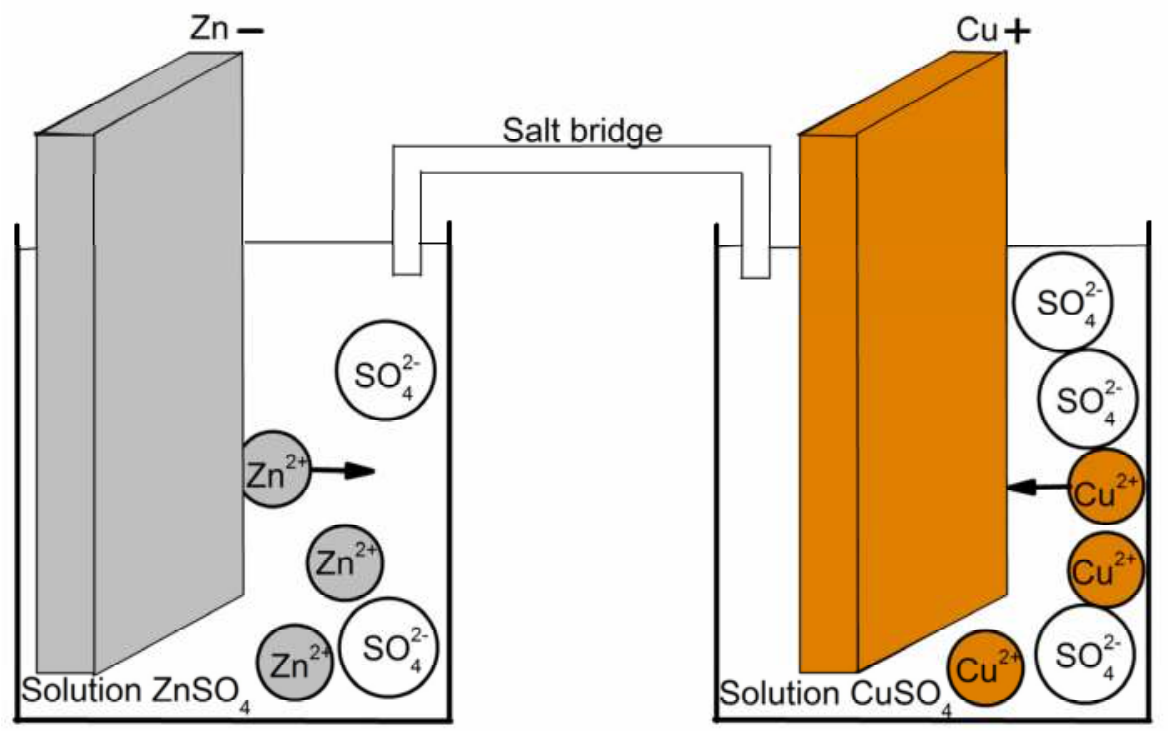

Figure 3. Establishment of equilibrium on the electrodes 
The change in electrode enthalpy involves dissolution enthalpy and thermal energy consumption upon ion transport into solution. The equation for dissolution enthalpy is readily obtained from eq. (16)

$$
\Delta_{\mathrm{r}} H_{1}=\Delta_{\mathrm{r}} H_{1}^{\mathrm{o}}-R T^{2} \frac{\partial \ln a_{1}}{\partial T}
$$

where $\Delta_{\mathrm{r}} H_{1}^{\mathrm{o}}$ is a standard change in enthalpy during ion dissolving. The total change in the enthalpy of the first electrode $\left(\Delta H_{1 \text {,thermostat }}\right.$ ) is the sum of the expressions $-\Delta_{\mathrm{r}} H_{1}$ and $-w_{\mathrm{g} 1}$

$$
\Delta H_{1, \text { thermostat }}=-\Delta_{\mathrm{r}} H_{1}-w_{\mathrm{g} 1}=-T \Delta_{\mathrm{r}} S_{1}^{\mathrm{o}}+R T \ln a_{1}+R T^{2} \frac{\partial \ln a_{1}}{\partial T}=-T \Delta_{\mathrm{r}} S_{1},
$$

where $\Delta_{\mathrm{r}} S_{1}^{\mathrm{O}}$ is a standard change in entropy upon ion dissolving and $\Delta_{\mathrm{r}} S_{1}$ is the change in entropy upon ion dissolving on the first electrode which amounts to

$$
\Delta_{\mathrm{r}} S_{1}=\Delta_{\mathrm{r}} S_{1}^{\mathrm{o}}-R \ln a_{1}-R T \frac{\partial \ln a_{1}}{\partial T} .
$$

As follows from eq. (22), the change in enthalpy, related to the first electrode, is independent of the processes, occurring on the second one. Therefore, studying either release or absorption of heat on a separate electrode, one may calculate the change in entropy due to the escape of the ions of the same type into the bulk.

A corresponding expression for the second electrode is of the same form but index "1" should be substituted by index "2":

$$
\begin{gathered}
\Delta_{\mathrm{r}} G_{2}=\Delta_{\mathrm{r}} G_{2}^{\mathrm{o}}+R T \ln a_{2}, \\
w_{2 g}=n F \Delta_{\mathrm{Met} 2}^{\mathrm{sol}} \varphi_{2}=-\Delta_{\mathrm{r}} G_{2}^{\mathrm{o}}-R T \ln a_{2} \\
\Delta_{\mathrm{Met} 2}^{\mathrm{sol}} \varphi_{2}=-\frac{\Delta_{\mathrm{r}} G_{2}^{\mathrm{o}}}{n F}-\frac{R T \ln a_{2}}{n F} . \\
\Delta_{\mathrm{r}} H_{2}=\Delta_{\mathrm{r}} H_{2}^{\mathrm{o}}-R T^{2} \frac{\partial \ln a_{2}}{\partial T}, \\
\Delta_{\mathrm{r}} S_{2}=\Delta_{\mathrm{r}} S_{2}^{\mathrm{o}}-R \ln a_{2}-R T \frac{\partial \ln a_{2}}{\partial T}, \\
\Delta H_{2, \text { thermostat }}=-\Delta_{\mathrm{r}} H_{2}-w_{2 g}=-T \Delta_{\mathrm{r}} S_{2}^{\mathrm{o}}+R T \ln a_{2}+R T^{2} \frac{\partial \ln a_{2}}{\partial T}=-T \Delta_{\mathrm{r}} S_{2} .
\end{gathered}
$$

In the operation of the galvanic cell, the processes on the second electrode are oppositely directed which should be taken into account in consideration of the thermodynamic cell parameters. 
Equations (20) and (26) allow to get the Nernst equation for the potential of the cell [3]

$$
E=-\Delta_{\text {Met2 }}^{\mathrm{sol}} \varphi_{2}+\Delta_{\text {Met1 }}^{\mathrm{sol}} \varphi_{1}=-\frac{\Delta_{\mathrm{r}} G^{\mathrm{o}}}{n F}-\frac{R T}{n F} \ln \frac{a_{1}}{a_{2}},
$$

where $E$ - the cell potential, $\Delta_{\mathrm{r}} G^{\mathrm{o}}=\Delta_{\mathrm{r}} G_{1}^{\mathrm{o}}-\Delta_{\mathrm{r}} G_{2}^{\mathrm{o}}$.

The electric work of the galvanic cell $\left(w_{\mathrm{el}}\right)$ results from the transformation of the potential energy of the charged electrodes into electric energy. The potential energy arises from the thermal energy of both of the electrodes upon ions transport into solution and equals

$$
w_{\mathrm{el}}=w_{1 g}-w_{2 g}=-\Delta_{\mathrm{r}} G^{\mathrm{o}}-R T \ln \frac{a_{1}}{a_{2}}=-w_{\mathrm{useful}} .
$$

The change in thermostat enthalpy is of the form

$$
\Delta H_{\text {thermostat }}=\Delta H_{1, \text { thermostat }}-\Delta H_{2, \text { thermostat }}=-\mathrm{T} \Delta_{\mathrm{r}} S \text {, }
$$

which is in fair agreement with similar expression, described by eq. (14), for the VHEB. The detailed equation for $\Delta H_{\text {thermostat }}$ can be get after substitution corresponding expressions (22) and (29) into (32)

$$
\Delta H_{\text {thermostat }}=-T\left(\Delta_{\mathrm{r}} S_{1}^{\mathrm{o}}-\Delta_{\mathrm{r}} S_{2}^{\mathrm{o}}\right)-R T \ln \left(a_{2} / a_{1}\right)-R T^{2}\left(\frac{\partial \ln a_{2}}{\partial T}-\frac{\partial \ln a_{1}}{\partial T}\right) .
$$

The sum of eqs. (31) and (33) gives the total energy (electric work + heat), produced by the galvanic cell

$$
w_{\mathrm{el}}+\Delta H_{\text {thermostat }}=-\Delta_{\mathrm{r}} H^{\mathrm{o}}-R T^{2}\left(\frac{\partial \ln a_{2}}{\partial T}-\frac{\partial \ln a_{1}}{\partial T}\right) .
$$

From eq.(34) it follows that the total energy produced by the galvanic cell is equal to the heat emitted by oxidation-reduction reaction.

Thus, the approach, based on the analysis of the behavior of one ion gives the same results as the present-day theory. However, it uses not a mysterious, direct transformation of the chemical energy $\left(\Delta_{\mathrm{r}} G\right)$ into electric work, but the concept of chemical energy conversion into the thermal one, and then, the thermal energy of thermostat (environment) is converted into the potential energy of charged electrodes $[12,13]$. The electric energy of the galvanic cell arises according to the scheme:

reaction heat $\rightarrow$ thermal thermostat energy $\rightarrow$ potential energy of charged electrodes $\rightarrow$ electric energy.

Thus, in various systems with uniform temperature, useful work is produced by the same mechanism through the exchange of thermal energy with thermostat (environment). No direct conversion of chemical energy into useful work is observed. Unfortunately, in the 
galvanic cell, the processes of heat release and useful work production cannot be spatially separated, because both of them occur in a double layer. Therefore, galvanic cells are unpromising in production of a double amount of energy.

\subsection{A concentration cell}

Consider now a concentration cell, consisting of two electrodes, e.g., the zinc ones of different solution activity. Standard changes in the Gibbs function, enthalpy, and entropy for the concentration cell tend to zero due to the same chemical nature of both of the electrodes. By definition, it has been considered that $a_{2}>a_{1}$. From eq. (31) it follows

$$
w_{\mathrm{el}}=R T \ln \left(a_{2} / a_{1}\right)=-w_{\mathrm{useful}},
$$

which is a usual expression for the electric energy of the concentration cell. From eq. (34) it follows

$$
w_{\mathrm{el}}+\Delta H_{\text {thermostat }}=-R T^{2}\left(\frac{\partial \ln a_{2}}{\partial T}-\frac{\partial \ln a_{1}}{\partial T}\right) .
$$

For the system in which the activities are temperature-independent, the electric energy arises from the thermal thermostat energy (environment)

$$
w_{\mathrm{el}}=-\Delta H_{\text {thermostat }}=-w_{\text {useful }},
$$

which is in fair agreement with conventional concepts.

\section{Useful work of the systems with concentration gradient}

An ideal system with concentration gradient has no potential energy because the mixing does not result in heat release and $\Delta H=0$. Nevertheless, the system with concentration gradient can be used, as any non-equilibrium system, to produce useful work if this system is supplied with special tools for extracting heat from the environment with a simultaneous transformation of the extracted thermal energy into work upon the system approaches to the equilibrium. The volume of useful work is equal to the volume of the heat extracted from the environment

$$
w_{\text {useful }}=\Delta H_{\text {thermostat }}
$$

The useful work of the system with a concentration gradient $w_{\text {useful }}$ obeys expressions (35) and (38). The concentration cell is a good example of such a system.

\section{General conclusions}

Any non-equilibrium state can serve as an energy source. The thermostat (environment) is an active participant of the process of reversible useful work production in devices operating at constant temperature. The heat released by chemical reactions, always 
dissipates in the thermostat (environment). The useful work is produced by special tools that provide the extraction of the thermal energy of the thermostat (environment) and the transformation of thermal energy into work at the process of the restoration of the chemical equilibrium. The volume of the useful work is equal, in reversible conditions, to the change in Gibbs function. A spatial separation of reactor and tools can lead to a substantial increase in the energy produced. The direct conversion of the Gibbs energy into useful work does not exist. The concepts of free and bound energy become unnecessary.

\section{Author details}

Nikolai Bazhin

Institute of Chemical Kinetics and Combustion, Novosibirsk State University, Institutskaya 3,

Novosibirsk, Russia

\section{References}

[1] Denbigh K (1971) The Principles of Chemical Equilibrium, 3rd ed., The University Press: Cambridge, $494 \mathrm{p}$.

[2] IUPAC Green Book (1996) Quantities, Units and Symbols in Physical Chemistry 48 p.

[3] Atkins P (2001) The Elements of Physical Chemistry, 3rd ed., Oxford University Press, Oxford, $.549 \mathrm{p}$.

[4] Lower S. (2010) Chemistry. Available http://www.chem1.com/acad/webtext/ virtualtextbook.html

[5] Gokcen NA, Reddy RG (1996) Thermodynamics, Second edition, Springer, 416 p.

[6] Haynie DN (2008), Biological Thermodynamics, $2^{\text {nd }}$ ed., Cambridge University Press, $422 \mathrm{p}$.

[7] Haywood RW (1980) Equilibrium Thermodynamics for Engineers and Scientists, J. Wiley, N.Y., 456 p.

[8] Adam NK (1956) Physical Chemistry, Oxford, Clarendon Press, 658 p.

[9] Physical Chemistry (1980) Gerasimov YaI, Ed., Chimiya, Moscow (in Russian), 1279 p.

[10] Steiner LE (1948) Introduction to Chemical Thermodynamic, 2nd ed.; McGraw-Hill Book Company, New York, 516 p.

[11] Bazhin NM, Parmon VN (2007) Conversion of the Chemical Reaction Energy into Useful Work in the Van't Hoff Equilibrium Box. J. Chem. Ed. 84: 1053 - 1055

[12] Bazhin NM, Parmon VN (2007) Ways of Energy Conversion in Electrochemical Cells. Doklady Physical Chemistry 417: 335-336

[13] Bazhin NM (2011) Mechanism of electric energy production in galvanic and concentration cells. J. Eng. Thermophysics 20: 302-307 